Mathematical Research Letters 12, 633-654 (2005)

\title{
ANCIENT SOLUTIONS TO KÄHLER-RICCI FLOW
}

\author{
LEI NI
}

\begin{abstract}
In this paper, we prove that any non-flat ancient solution to KählerRicci flow with bounded nonnegative bisectional curvature has asymptotic volume ratio zero. We also classify all complete gradient shrinking solitons with nonnegative bisectional curvature. Both results generalize the corresponding earlier results of Perelman in [P1] and [P2]. The results then are applied to study the geometry and function theory of complete Kähler manifolds with nonnegative bisectional curvature via Kähler-Ricci flow. A compactness result on ancient solutions to Kähler-Ricci flow is also obtained.
\end{abstract}

\section{Introduction}

The Kähler-Ricci flow

$$
\frac{\partial}{\partial t} g_{\alpha \bar{\beta}}(x, t)=-R_{\alpha \bar{\beta}}(x, t)
$$

has been useful in the study of complex geometry in the work of [B, C1, M2], etc. The ancient solution, a solution defined for $-\infty<t \leq 0$, arises [H5] when one applies the parabolic blow-up to a finite time singularity or slowly forming (Type II) singularity as $t$ approaches infinity. In [H5] Hamilton introduced some geometric invariants associated with ancient solutions. One of them is the so-called asymptotic volume ratio (also called cone angle at infinity), which is defined as $\mathcal{V}(M, g):=\lim _{r \rightarrow \infty} \frac{V_{o}(r)}{\omega_{n} r^{n}}$, for any complete Riemannian manifold $(M, g)$. Here $n$ is the (real) dimension of $M, V_{o}(r)$ is the volume of the ball of radius $r$ centered at $o$ (with respect to metric $g$ ) and $\omega_{n}$ is the volume of unit ball in $\mathbb{R}^{n}$. This asymptotic volume ratio is well-defined and independent of the choice of $o$ in the case when $M$ has non-negative Ricci curvature, by the Bishop volume comparison. One should consult [H5] for the condition (and the proof) under which $\mathcal{V}(M, g(t))$ is independent of $t$ for a family of metrics $g(t)$ satisfying Ricci flow. When the meaning is clear in the context we simply denote $\mathcal{V}(M, g(t))$ by $\mathcal{V}(g(t))$.

In [P1], Perelman studied the properties of ancient solutions with bounded nonnegative curvature operator, via his entropy and reduced volume (reduced distance) monotonicity formulae. In particular, the following result is proved.

Received by the editors August 8, 2004.

Research partially supported by NSF grants and an Alfred P. Sloan Fellowship, USA. 
Theorem 1. Let $(M, g(t))$ be a complete non-flat ancient solution to Ricci flow, with bounded nonnegative curvature operator. Then $\mathcal{V}(g(t))=0$.

This result holds the key to the rest striking results on ancient solutions in [P1]. The assumption on the nonnegativity of the curvature operator is ensured in dimension three by Hamilton-Ivey's pinching estimate [H5, Theorem 24.4], if the ancient solution is obtained from the blow-up limit of a finite time singularity. It is also needed to make effective uses of the reduced distance introduced in [P1, Section 7] in Perelman's blow down procedure [P1. Section 11] on the ancient solutions. For Kähler-Ricci flow, one would like to replace the nonnegativity of the curvature operator by the nonnegativity of bisectional curvature since the nonnegativity of the sectional curvature is neither natural nor necessarily preserved under Kähler-Ricci flow. On the other hand, the argument of [P1, Section 11] made essential uses of the nonnegativity of sectional curvature and the properties of such Alexandrov spaces. There is no obvious way of adapting the proof of [P1, Section 11.4] to the Kähler setting. Therefore one needs some new ingredients in order to generalize Perelman's result to KählerRicci flow assuming only the nonnegativity of the bisectional curvature. It turns out that this technical hurdle can be overcome by a result (Proposition 1.1) on the shrinking solitons, Perelman's blow-down procedure and a splitting result for Kähler manifolds with nonnegative bisectional curvature, proved recently by Luen-Fai Tam and the author in [NT2]. These will be the main focus of this paper.

There exists another motivation of proving Theorem 1 for Kähler-Ricci flow on Kähler manifolds with nonnegative bisectional curvature. In a AMS meeting (November 2001, held at Irvine, California), Huai-dong Cao proposed the following conjecture (see also [C4] for a related problem on ancient solutions).

Conjecture (Cao). Let $\left(M^{m}, g(t)\right)$ be a non-flat steady gradient Kähler-Ricci soliton with bounded nonnegative bisectional curvature. Then $\mathcal{V}(g(t))=0$.

Proving Theorem 1 for Kähler-Ricci flow on Kähler manifolds with nonnegative bisectional curvature will imply Cao's conjecture. Combining the techniques from $[\mathrm{P} 1]$ and $[\mathrm{P} 2]$ with [NT2], we indeed can prove the following such generalization of Theorem 1.

Theorem 2. Let $\left(M^{m}, g(t)\right)\left(m=\operatorname{dim}_{\mathbb{C}}(M), n=2 m\right)$ be a non-flat ancient solution to Kähler-Ricci flow (0.1). Assume that $(M, g(t))$ has bounded nonnegative bisectional curvature. Then $\mathcal{V}(g(t))=0$.

The only known result along this line, under the assumption of nonnegative bisectional curvature has been only proved for the case of $m=2$, and only for the gradient Kähler-Ricci solitons, which are special ancient solutions. Please see, for example [C4, CZ2]. Following [P1], an immediate consequence of Theorem 2 is the following compactness result.

For an fixed $\kappa>0$, the set of $\kappa$-solution to Kähler-Ricci flow is compact module scaling. 
Please refer to Section 2 for the definitions of the $\kappa$-solutions. A compactness result for the elliptic case, namely the Kähler-Einstein metrics, was proved earlier in $[\mathrm{T}]$ for compact manifolds under extra assumptions on an integral bound of curvature, a volume lower bound and a diameter upper bound.

Since for the Riemannian manifolds with non-negative Ricci curvature, the Bishop volume comparison theorem asserts that $\frac{V_{o}(r)}{\omega_{2 m} r^{2 m}}$ is monotone non-increasing, the manifold $M$ is called of maximum volume growth if $\mathcal{V}(M, g)>0$. The above result simply states that the ancient solutions with bounded nonnegative bisectional curvature (which is preserved under the KählerRicci flow, by [B, M2]) can not be of maximum volume growth. Applying Shi's short time existence result [Sh1], Hamilton's singularity analysis argument in [H5, Theorem 16.2] (or Perelman's result in [P1, Section 11]), as well as estimates from [NT1] and [N2] one can have the following corollary of Theorem 2 .

Corollary 1. Let $\left(M^{m}, g_{0}\right)\left(m=\operatorname{dim}_{\mathbb{C}}(M)\right)$ be a complete Kähler manifold with bounded nonnegative bisectional curvature. Assume that $M$ is of maximum volume growth. Then the Kähler-Ricci flow $(0.1)$, with $g(x, 0)=g_{0}(x)$ has a long time solution. Moreover the solution has no slowly forming (Type II) singularity as $t$ approaches $\infty$. In particular, there exists a $C=C\left(M, g_{0}\right)>0$ such that the scalar curvature $\mathcal{R}(y)$ satisfies

$$
f_{B_{x}(r)} \mathcal{R}(y) d \mu \leq \frac{C}{(1+r)^{2}}
$$

where $B_{x}(r)$ is the ball of radius $r$ centered at $x, V_{x}(r)=\operatorname{Vol}\left(B_{x}(r)\right)$ and $f_{B_{x}(r)} f(y) d \mu=\frac{1}{V_{x}(r)} \int_{B_{x}(r)} f(y) d \mu$. Furthermore, $M$ is diffeomorphic (homeomorphic) to $\mathbb{C}^{m}$, for $m>2(m=2)$, and is biholomorphic to a pseudoconvex domain in $\mathbb{C}^{m}$.

The curvature decay statement of Corollary 1 confirms a conjecture of Yau in [Y, page 621], where he speculated that two assumptions in Shi's main theorem of [Sh3] can be replaced by the maximum volume growth alone. See also the recent work of $\mathrm{Wu}$ and Zheng [WuZ] on various examples related to the above corollary. Corollary 1 also provides a partial answer to a question asked in [N2, Conjecture 3.1] on conditions equivalent to the existence of holomorphic functions of polynomial growth. In fact, there the author speculated that either the maximum volume growth or the average quadratic curvature decay is equivalent to the existence of nonconstant polynomial growth holomorphic functions provided the manifold has quasi-positive bisectional curvature. There also exists a related general conjecture of Yau on the non-existence of the bounded holomorphic functions, on which one can refer to [LW] for some recent progresses. Using Perelman's Theorem 1 one can conclude the similar result for the Riemannian manifolds with bounded nonnegative curvature operator and maximum volume growth. For Kähler-Ricci flow, under the stronger assumption that $M$ is 
a Kähler manifold with bounded nonnegative curvature operator and maximum volume growth, a similar (but slightly weaker) estimate as (0.2) was proved earlier in [CZ4] by applying the dimension reduction argument of Hamilton. This is in fact a fairly easy consequence of Perelman's Theorem 1. (See the proof of Corollary 1 for details. The main constraint of related results in [H5], for the application to Kähler geometry, is that the argument of [H5] only works under the stronger assumption on nonnegativity of curvature operator/sectional curvature, which is sufficient for the study of three manifolds, but not for the study of Kähler manifolds of complex dimension $\geq 3$.) In [Sh3-4], the long time existence result was proved under a uniform curvature decay assumption similar to (0.2), with/without the assumption of the maximum volume growth. In Corollary 1 we have the curvature decay as a consequence instead of an assumption, exactly as what Yau speculated in $[Y]$. Under certain further curvature average decay assumptions, the topological conclusion in Corollary 1 was proved earlier in [CZ3]. (See also [H6, Sh4] for related earlier fundamental works. The key observation on the improvement of injectivity radius lower bound was first observed in [Sh4].) The last statement of Corollary 1 was proved first in [Sh4] (see also [CZ3]) under the assumptions that the manifold has positive sectional curvature and a certain curvature average decay condition. Note also that Corollary 4.1 of [NT2] proved that $M$ is Stein if $M$ has maximum volume growth and nonnegative bisectional curvature (which is a conjecture of $\mathrm{H}$. Wu).

Corollary 2. Let $\left(M^{m}, g_{0}\right)$ be a complete Kähler manifold with bounded nonnegative holomorphic bisectional curvature and maximum volume growth. Then the transcendence degree of the rational function field of $M$ (see [N2] for definition) is equal to $m$. Furthermore, $M$ is biholomorphic to an affine quasi-algebraic variety. When $m=2, M$ is biholomorphic to $\mathbb{C}^{2}$.

In [M1, M3], a systematic scheme on embedding/compactifying complete Kähler manifolds with positive curvature was developed. One can also consult the survey article [M4] for expositions on these methods and related results. Originally in [M1] under the assumption that $M$ has positive bisectional curvature and certain curvature decay conditions, the conclusion that $M$ is biholomorphic to $\mathbb{C}^{2}$ in the case $m=2$, and that $M$ is biholomorphic to an affine variety in the higher dimension were obtained. In fact in his fundamental work [M1] Mok laid down the framework and basic line of argument of the affine embedding and observed that one can apply the result of Ramanujam, which asserts that a quasi-projective surface homeomorphic to $\mathbb{R}^{4}$ must be biholomorphic to $\mathbb{C}^{2}$, in the case $m=2$. Later, for the case $m=2$ only, in [CTZ], following Mok's compactification scheme in [M1. M3], the authors improved the above result of Mok, replacing the assumptions of [M1] by that $M$ has bounded positive bisectional curvature and of maximum volume growth. The result stated in [CTZ] also assumes a mild average curvature decay condition which replaces the stronger point-wise curvature decay assumption of [M1] via Kähler-Ricci flow. This average curvature decay condition can be removed by combining with another later 
paper [CZ4], again assuming the positivity of the bisectional curvature and volume being of maximum growth. In this later improvement [CTZ], which is only restricted to the case of $m=2$ (also the earlier paper [CZ2]), the proof also crucially relies on an observation only valid in complex dimension two, originally due to Ivey [I], that an ancient solution to Kähler-Ricci flow with nonnegative bisectional curvature must have nonnegative curvature operator. Namely the method there relied crucially on the dimensional reduction results of Hamilton in [H5]. Hence it does not work under the assumption of the nonnegativity of bisectional curvature in higher dimensions.

In Corollary 2, when $m=2$, our statement assumes only nonnegativity instead of positivity of the bisectional curvature. Moreover, our new approach also works for the higher dimensional case. Note that the uniform multiplicity estimates recently proved in [N2] simplifies the steps in [M1] quite a bit. We should point out that the $m=2$ case can also be obtained by combining Corollary 4.1 of [NT2] with the argument of [CTZ]. However the method of current paper provides an unified direct approach which works also for higher dimensions.

In the proof of Theorem 2 we need the following result on gradient shrinking solitons of Kähler-Ricci flow, which is of independent interests.

Theorem 3. Let $\left(M^{m}, g\right)$ be a non-flat gradient shrinking soliton to KählerRicci flow.

(i) If the bisectional curvature of $M$ is positive then $M$ must be compact and isometric-biholomorphic to $\mathbb{P}^{m}$.

(ii) If $M$ has nonnegative bisectional curvature then the universal cover $\tilde{M}$ splits as $\tilde{M}=N_{1} \times N_{2} \times \cdots \times N_{l} \times \mathbb{C}^{k}$ isometric-biholomorphically, where $N_{i}$ are compact irreducible Hermitian Symmetric Spaces.

In particular, $\mathcal{V}(M, g)=0$.

Theorem 3 generalizes a corresponding recent result of [P2, Lemma 1.2], where Perelman shows that in dimension three, any $\kappa$-noncollapsed gradient shrinking soliton with bounded positive sectional curvature must be compact. When $M$ is compact and $m=2$, the classification in part (ii) was obtained in [I] under even weaker assumption on the nonnegativity of the isotropic curvature.

For recent works on Kähler-Ricci flow on compact manifolds, one should refer to the survey articles [CC, Cn]. Perelman [P3] also has some important work on the conjecture (which arises from the related works of Hamilton and Tian) concerning the large time behavior of (normalized) Kähler-Ricci flow on compact Kähler manifolds with $c_{1}(M)>0$ (cf. [N3]). In [CT1-2], very recent progresses (and further applications of Corollary 1) have been made towards the uniformization problem addressed in Corollary 2.

Finally, we should point out that when dimension $m=1$ the above results are known from the work of Hamilton [H3] and [H5, Section 26]. (See also [Ch] and $[\mathrm{CK}, \mathrm{CLN}]$.

The method of this paper has other applications in the study of Ricci flow on Riemannian manifolds. Please see Section 3. 


\section{Proof to Theorem 2 and 3}

Recall that a complete Riemannian manifold $(M, g)$ is called a gradient shrinking soliton if there exists a smooth function $f$ such that, for some positive constant $a$,

$$
\nabla_{i} \nabla_{j} f+R_{i j}-a g_{i j}=0 .
$$

The following result generalizes the result of Perelman by removing the uniform curvature bound.

Proposition 1.1. Let $(M, g)$ be a Ricci non-flat gradient shrinking soliton. Assume that the Ricci curvature of $M$ is nonnegative. Then there exists a $\delta=\delta(M)(1 \geq \delta>0)$ such that

$$
\mathcal{R}(x) \geq \delta>0 .
$$

Proof. It is easy to see, from the strong maximum principle, that the scalar curvature $\mathcal{R}(x)>0$. Differentiating (1.1) and applying the second Bianchi identity, we have that

$$
\nabla_{i} \mathcal{R}=2 R_{i j} f_{j}
$$

This implies that

$$
\nabla_{i} \mathcal{R}+2 f_{i j} f_{j}-2 a f_{i}=2\left(R_{i j}+f_{i j}-a g_{i j}\right) f_{j}=0
$$

which further implies that there exists a constant $C_{1}=C_{1}(M)$ such that

$$
\mathcal{R}+|\nabla f|^{2}-2 a f=C_{1} .
$$

These equations are well known for the gradient shrinking solitons.

Let $o \in M$ be a fixed point. For any $x \in M$ we denote the distance of $x$ from $o$ by $r(x)$. Let $\gamma(s)$ be minimal geodesic joining $x$ from $o$, parametrized by the arc-length. For simplicity we often also denote $r(x)$ by $s_{0}$. Let $\left\{E_{i}(s)\right\}$ $(0 \leq i \leq n-1)$ be a parallel transplanted orthonormal frame along $\gamma(s)$ such that $E_{0}(s)=\gamma^{\prime}(s)$. If $s_{0} \geq 2$, for $s_{0} \geq r_{0} \geq 1$, define $n-1$-variational vector fields $Y_{i}(s)(1 \leq i \leq n-1)$ along $\gamma(s)$ by

$$
Y_{i}(s)=\left\{\begin{array}{l}
s E_{i}(s), \quad 0 \leq s \leq 1 \\
E_{i}(s), \quad 1 \leq s \leq s_{0}-r_{0} \\
\frac{s_{0}-s}{r_{0}} E_{i}(s), s_{0}-r_{0} \leq s \leq s_{0}
\end{array} .\right.
$$

From the second variation consideration [P1, Lemma 8.3 (b)] (see also [H5, Theorem 17.4]), we have that

$$
\sum_{i=1}^{n-1} \int_{0}^{s_{0}}\left|Y_{i}^{\prime}(s)\right|^{2}-R\left(\gamma^{\prime}(s), Y_{i}(s), \gamma^{\prime}(s), Y_{i}(s)\right) d s \geq 0 .
$$


In particular we can find $C(M)$, which depends only the upper bound of the Ricci curvature of $M$ on $B_{o}(1)$, such that

$$
\begin{aligned}
\int_{0}^{s_{0}-r_{0}} \operatorname{Ric}\left(\gamma^{\prime}(s), \gamma^{\prime}(s)\right) d s & \leq C(M)+\frac{n-1}{r_{0}}-\int_{s_{0}-r_{0}}^{s_{0}}\left(\frac{s_{0}-s}{r_{0}}\right)^{2} \operatorname{Ric}\left(\gamma^{\prime}(s), \gamma^{\prime}(s)\right) d s \\
& \leq C(M)+\frac{n-1}{r_{0}}
\end{aligned}
$$

Here we have used the fact that the Ricci curvature is nonnegative. We claim that there exists a positive constant $A=A(M)$, if $s_{0} \geq A$ and $\mathcal{R}(x) \leq 1$, there exists another constant, still denoted by $C(M)$, such that

$$
\int_{0}^{s_{0}} \operatorname{Ric}\left(\gamma^{\prime}(s), \gamma^{\prime}(s)\right) d s \leq \frac{a}{2} s_{0}+C(M)
$$

Assume that we have proved the claim (1.6). Then there exists $C_{2}=C_{2}(M)>0$ such that

$$
\begin{aligned}
\left.\left\langle\nabla f, \gamma^{\prime}(s)\right\rangle\right|_{o} ^{\gamma(r(x))} & =\int_{0}^{s_{0}} \frac{d}{d s}\left(\left\langle\nabla f, \gamma^{\prime}(s)\right\rangle\right) d s \\
& =\int_{0}^{s_{0}}\left(\nabla_{i} \nabla_{j} f\right) \frac{d \gamma^{i}(s)}{d s} \frac{d \gamma^{j}(s)}{d s} d s \\
& =\int_{0}^{s_{0}}\left(a-\operatorname{Ric}\left(\gamma^{\prime}(s), \gamma^{\prime}(s)\right)\right) d s \\
& =a r(x)-\int_{0}^{s_{0}} \operatorname{Ric}\left(\gamma^{\prime}(s), \gamma^{\prime}(s)\right) d s \\
& \geq \frac{a}{2} r(x)-C_{2},
\end{aligned}
$$

which implies that for every $x \in M \backslash B_{o}(A)$ with $\mathcal{R}(x) \leq 1$,

$$
\langle\nabla f, \nabla r\rangle(x) \geq \frac{a}{2} r(x)-C_{2}-|\nabla f|(o) .
$$

It in particular implies that for every such $x$, with $r(x) \geq \frac{4}{a}\left(C_{2}+|\nabla f|(o)\right)$, $\nabla f(x) \neq 0$.

Now we can prove the proposition after the claim (1.6). For any $x \in M \backslash$ $\left(B_{o}(A) \cup B_{o}\left(\frac{4}{a}\left(C_{2}+|\nabla f|(o)\right)\right)\right)$, without the loss of generality we can assume that $\mathcal{R}(x) \leq 1$, let $\sigma(\eta)$ be the integral curves of $\nabla f$, passing $x$ with $\sigma(0)=x$. By (1.3) we have that

$$
-\frac{d}{d \eta}\left(\mathcal{R}(\sigma(\eta))=-2 R_{i j} \frac{d \sigma^{i}}{d \eta} \frac{d \sigma^{j}}{d \eta} \leq 0\right.
$$


This implies that $\mathcal{R}(x) \geq \mathcal{R}(\sigma(\eta))$, for $\eta<0$. On the other hand,

$$
-\frac{d}{d \eta} r(\sigma(\eta))=-\langle\nabla r, \nabla f\rangle \leq-\left(C_{2}+|\nabla f|(o)\right) \leq 0
$$

as far as $r(\sigma(\eta)) \geq \max \left(A, \frac{4}{a}\left(C_{2}+|\nabla f|(o)\right)\right)$, noticing that we always have $\mathcal{R}(\sigma(\eta)) \leq 1$. This implies that the integral curve $\sigma$ exists for all $\eta<0$ since $|\nabla f|$ is bounded inside the closed ball $\overline{B_{o}(2 r(x))}$. The estimate (1.8) also implies that there exists $\eta_{1}<0$ such that $r\left(\sigma\left(\eta_{1}\right)\right)=\max \left(A, \frac{4}{a}\left(C_{2}+|\nabla f|(o)\right)\right)$. Applying (1.7) we have that

$$
\mathcal{R}(x) \geq \inf _{y \in B_{o}\left(r\left(\sigma\left(\eta_{1}\right)\right)\right)} \mathcal{R}(y) .
$$

This proves the proposition assuming the claim (1.6).

Now we prove the claim (1.6). First by equation (1.3) and the fact $R_{i j} \geq 0$ we have that $f_{i j} \leq a g_{i j}$. This implies that along any minimizing geodesic $\gamma(s)$ from $o, f^{\prime \prime}(s) \leq a$. Hence there exists $B=B(M)$ such that

$$
f(x) \leq(a+1) r^{2}(x)
$$

for $r(x) \geq B$. Using (1.4) and the fact that $\mathcal{R}>0$ we have that

$$
|\nabla f|(x) \leq 2(a+1) r(x)
$$

for $r(x) \geq B$. On the other hand, (1.3) also implies that

$$
|\nabla \mathcal{R}|^{2} \leq 4 \mathcal{R}^{2}|\nabla f|^{2}
$$

The above two inequality implies the the estimate

$$
|\nabla \log \mathcal{R}|(x) \leq 2(a+1) r(x)
$$

for $r(x) \geq B$. Now we adapt the notations and situations right before (1.6) and choose $r_{0}$ in (1.5) such that $\frac{n-1}{r_{0}}=\epsilon s_{0}$ with some fixed positive constant $\epsilon \leq \min \left(1, \frac{a}{2}\right)$. Then (1.6) implies that

$$
\int_{0}^{s_{0}-r_{0}} \operatorname{Ric}\left(\gamma^{\prime}(s), \gamma^{\prime}(s)\right) d s \leq C(M)+\epsilon s_{0}
$$

Notice $r_{0}=\frac{n-1}{\epsilon s_{0}} \leq \frac{n-1}{\epsilon} \leq \frac{s_{0}}{2}$ if $s_{0} \geq A$ for some $A=A(M) \geq \max \left(1,2 B, 2 \frac{n-1}{\epsilon}\right)$. Now using the gradient estimate on $\log \mathcal{R}$ above we have that

$$
\begin{aligned}
\log \frac{\mathcal{R}\left(\gamma\left(s_{1}\right)\right)}{\mathcal{R}\left(\gamma\left(s_{0}\right)\right)} & =-\int_{s_{1}}^{s_{0}} \frac{d}{d s} \log \mathcal{R}(\gamma(s)) d s \\
& \leq \int_{s_{1}}^{s_{0}}|\nabla \log \mathcal{R}| d s \\
& \leq 2(a+1) s_{0}\left(s_{0}-s_{1}\right)
\end{aligned}
$$


for $s_{1} \leq s_{0}$. Hence

$$
\mathcal{R}(\gamma(s)) \leq \mathcal{R}\left(\gamma\left(s_{0}\right)\right) \exp \left(\frac{2(a+1)(n-1)}{\epsilon}\right) \leq \exp \left(\frac{2(a+1)(n-1)}{\epsilon}\right)
$$

for any $s \geq s_{0}-r_{0}$. Here we have used the assumption $\mathcal{R}(x)=\mathcal{R}\left(\gamma\left(s_{0}\right)\right) \leq 1$. This further implies that

$$
\begin{aligned}
\int_{s_{0}-r_{0}}^{s_{0}} \operatorname{Ric}\left(\gamma^{\prime}(s), \gamma^{\prime}(s)\right) d s & \leq \int_{s_{0}-r_{0}}^{s_{0}} \mathcal{R}(\gamma(s)) d s \\
& \leq r_{0} \exp \left(\frac{2(a+1)(n-1)}{\epsilon}\right) \\
& =\frac{n-1}{\epsilon s_{0}} \exp \left(\frac{2(a+1)(n-1)}{\epsilon}\right) \\
& \leq C(\epsilon, M) .
\end{aligned}
$$

Together with (1.9), we prove our claim (1.6). Hence we complete the proof of the Proposition 1.1.

Proof of Theorem 3. Case (i). By the strong maximum principle we can assume that $\mathcal{R}>0$ everywhere, otherwise one would have that $M$ is Ricci flat, hence flat. By Proposition 1.1, we know that $\mathcal{R} \geq \delta>0$, for some $\delta$. In particular, it implies that for any $x$

$$
f_{B_{x}(r)} \mathcal{R}(y) d \mu \geq \delta
$$

Here $f$ is the average integral defined in Corollary 1 of the introduction. On the other hand, part (ii) of Theorem 4.2 in [NT2] implies that if $M$ is not compact, then it must satisfies

$$
f_{B_{x}(r)} \mathcal{R}(y) d \mu \leq \frac{C_{2}}{1+r}
$$

for some $C_{2}(x, M)>0$. This is a contradiction! This implies that $M$ must be compact.

Case (ii). Let $\tilde{M}$ be the universal cover of $M$. Since $\tilde{M}$ is still a shrinking soliton, we can apply Proposition 1.1 to $\tilde{M}$. From part (i) of Theorem 4.2 in [NT2], we can split $\tilde{M}$ as a product of two manifolds with one of the factor being compact and the other satisfies the curvature average decay (1.10). The classification result follows from the celebrated results of Siu-Yau [SiY] (also due to Mori), Mok [M2] as well as an observation of Koiso [Ko, Proposition 1.3].

The conclusion $\mathcal{V}(M, g)=0$ now follows easily from (i) and (ii).

The proof of Theorem 2 requires the blow-down procedure of Perelman in [P1, Proposition 11.2]. Since Proposition 11.2 of [P1] provided only a sketched 
proof, in the following we present in a more detailed way the blow-down procedure of Perelman of $[\mathrm{P} 1,11.2]$ on the so-called bounded $\kappa$-solutions, which are defined to be complete ancient solutions with bounded nonnegative bisectional curvature and $\kappa$-noncollapsed in all scales. (See also [CLN], [KL], [STW] and $[\mathrm{Ye}]$ for various expositions on this part.) A solution (to Ricci flow) is called $\kappa$-noncollapsed if for any time $t$, for any ball $B_{x}(r)$ (with respect to metric $g(t)$ ), satisfying $|R m|(y) \leq \frac{1}{r^{2}}$ for all $y \in B_{x}(r)$ one has $V_{x}(r) \geq \kappa r^{n}$. One should refer to [P1, Section 4] for more discussions on $\kappa$-noncollapsing, [P1, Section 7, 11] for more details of the reduced distance and its properties. Now we adapt [P1, Section 7,11 ] to Kähler-Ricci flow and replace the nonnegativity of the curvature operator by the nonnegativity of the bisectional curvature. So in the following, we let $(M, g(t))$ be a complete non-flat ancient solution to Kähler-Ricci flow. We also assume that $(M, g(t))$ has bounded nonnegative bisectional curvature (the boundedness of curvature can be replaced by the differential Harnack inequality, (1.14) below) and it is $\kappa$-non-collapsed for some $\kappa>0$.

First we recall the definition of the reduced distance $\ell(y, \tau)$. Fix a space-time point $\left(x_{0}, t_{0}\right)$. Let $\tau=t_{0}-t$. Define

$$
\ell(y, \tau)=\inf _{\gamma, \gamma(0)=x_{0}, \gamma(\tau)=y} \frac{1}{2 \sqrt{\tau}} \int_{0}^{\tau} \sqrt{\eta}\left(\mathcal{R}+4\left|\gamma^{\prime}(\eta)\right|^{2}\right) d \eta
$$

We have factor 4 here due to different convention in Kähler category. Since the $\mathcal{R} \geq 0$ and the metrics are shrinking (since $R_{\alpha \bar{\beta}} \geq 0$ ) along the $t$ direction it is easy to see that

$$
\ell(y, \tau) \geq \frac{d_{t_{0}}^{2}\left(y, x_{0}\right)}{\tau}
$$

(Sometimes we also denote $d_{t_{0}}\left(y, x_{0}\right)$ by $d_{0}\left(y, x_{0}\right)$ when we think in terms of $\tau$.) As a consequence of above lower bound on $\ell$ we know that $\ell(y, \tau)$ achieves its minimum for each $\tau$ at some point finite distance away from $x_{0}$. Thus we can conclude that

Claim 1. For each $\tau$ there exists a point $x(\tau)$ such that $\ell(x(\tau), \tau)=\frac{n}{2}$.

Proof. By the equation (7.15) in [P1], using the maximum principle it was shown in $[\mathrm{P} 1$, Section 7$]$ that $\min _{y \in M} \ell(y, \tau) \leq \frac{n}{2}$. Using the continuity and the above lower bound on $\ell(y, \tau)$ we know the existence of $x(\tau)$.

Let us recall the equations satisfied by $\ell(y, \tau)$ from [P1, Section 7]. First we have

$$
|\nabla \ell|^{2}+\mathcal{R}=\frac{1}{\tau} \ell-\frac{1}{\tau^{\frac{3}{2}}} K
$$

Here

$$
K=\int_{0}^{\tau} \eta^{\frac{3}{2}} H(X) d \eta
$$


where $H(X)=-\mathcal{R}_{\tau}-2\langle\nabla \mathcal{R}, X\rangle-2\langle X, \nabla \mathcal{R}\rangle+4 \operatorname{Ric}(X, X)-\frac{1}{\tau} \mathcal{R}$ is the trace different Harnack (also called Li-Yau-Hamilton) expression for shrinking solitons, $X$ is $(1,0)$ component of the tangent vector of the minimizing $\mathcal{L}$-geodesics. Notice that $\langle\cdot, \cdot\rangle$ is the Hermitian product with respect to the Kähler metric. We also have that

$$
|\nabla \ell|^{2}+\ell_{\tau}=-\frac{1}{2 \tau^{\frac{3}{2}}} K
$$

and

$$
\ell_{\tau}=\mathcal{R}-\frac{\ell}{\tau}+\frac{1}{2 \tau^{\frac{3}{2}}} K
$$

Applying H.-D. Cao's [C2] (in stead of Hamilton's) trace differential Harnack (also called Li-Yau-Hamilton inequality) for ancient solutions to Kähler-Ricci flow,

$$
-\mathcal{R}_{\tau}-2\langle\nabla \mathcal{R}, X\rangle-2\langle X, \nabla \mathcal{R}\rangle+4 \operatorname{Ric}(X, X) \geq 0
$$

we have that

$$
H(X) \geq-\frac{1}{\eta} \mathcal{R}
$$

Therefore we have that

$$
K \geq-\int_{0}^{\tau} \sqrt{\eta} \mathcal{R} d \eta \geq-2 \sqrt{\tau} \ell
$$

Applying the above lower bound to (1.11)-(1.13) we have that

$$
\begin{aligned}
& |\nabla \ell|^{2}+\mathcal{R} \leq \frac{3}{\tau} \ell \\
& |\nabla \ell|^{2}+\ell_{\tau} \leq \frac{\ell}{\tau}
\end{aligned}
$$

and

$$
\ell_{\tau} \geq \mathcal{R}-\frac{2}{\tau} \ell \geq-\frac{2}{\tau} \ell
$$

From (1.15), we have that

$$
\left|\nabla \ell^{\frac{1}{2}}\right|^{2} \leq \frac{3}{4 \tau}
$$

which implies that

$$
\ell^{\frac{1}{2}}(y, \tau) \leq \sqrt{\frac{n}{2}}+d_{\tau}(x(\tau), y) \sqrt{\frac{3}{4 \tau}} .
$$

Now we can deduce the following results on the reduced distance $\ell(y, \tau)$. 
Corollary 1.1. For $y \in B_{\tau}\left(x(\tau), \sqrt{\frac{\tau}{\epsilon}}\right)$,

$$
\ell(y, \tau) \leq\left(\sqrt{\frac{n}{2}}+\sqrt{\frac{3}{4 \epsilon}}\right)^{2} .
$$

Hence

$$
\ell(y, \eta) \leq 4\left(\sqrt{\frac{n}{2}}+\sqrt{\frac{3}{4 \epsilon}}\right)^{2} .
$$

for all $2 \tau \geq \eta \geq \frac{\tau}{2}$ and $y \in B_{\tau}\left(x(\tau), \sqrt{\frac{\tau}{\epsilon}}\right)$. And

$$
\tau \mathcal{R}(y, \eta) \leq 12\left(\sqrt{\frac{n}{2}}+\sqrt{\frac{3}{4 \epsilon}}\right)^{2} .
$$

Moreover, on $B_{\tau}\left(x(\tau), \sqrt{\frac{\tau}{\epsilon}}\right) \times\left(\delta \tau, \frac{1}{\delta} \tau\right)$ one has that

$$
\ell(y, \eta) \leq\left(\frac{1}{\delta}\left(\sqrt{\frac{n}{2}}+\sqrt{\frac{3}{4 \epsilon}}\right)\right)^{2} .
$$

and

$$
\tau \mathcal{R}(y, \eta) \leq 3\left(\frac{1}{\delta}\left(\sqrt{\frac{n}{2}}+\sqrt{\frac{3}{4 \epsilon}}\right)\right)^{2}
$$

The following corollary gives relation between the $\kappa$-constant in the definition of $\kappa$-noncollapsing and the lower bound of the reduced volume.

Corollary 1.2. There exists a constant $C_{1}(n)>0$ such that

$$
\tilde{V}(\tau) \geq e^{-C_{1}(n)} \kappa
$$

This in particular implies that $\lim _{\tau \rightarrow \infty} \tilde{V}(\tau)>0$. On the other hand, for any $\delta>0$, there exists a $\kappa>0$ so that if $\tilde{V}(\tau) \geq \delta>0$ for any $\tau>0$ and any $\left(x_{0}, t_{0}\right) \in M \times(-\infty, 0)$. Then $M \times(-\infty, 0)$ is $\kappa$-noncollapsed at all scales.

Proof. Apply the $(1.21)$ to $B_{\tau}(x(\tau), \tau)$ to see that it satisfies the curvature bound assumption on the ball in the non-collapsing assumption. Then the result follows from the estimate (1.20) and the definition of the reduced volume $\tilde{V}(\tau)=\int_{M} \frac{e^{-\ell(y \tau)}}{\tau^{\frac{n}{2}}} d \mu_{\tau}$. The converse follows from the second $\kappa$-noncollapsing proof of Perelman in [P, Section 7]. 
Let $\tilde{g}^{\tau}(s)=\frac{1}{\tau} \bar{g}(s \tau)$. Then $\tilde{B}_{1}\left(x(\tau), \sqrt{\frac{1}{\epsilon}}\right)=B_{\tau}\left(x(\tau), \sqrt{\frac{\tau}{\epsilon}}\right)$. The $\left(1.21^{\prime}\right)$ and the $\kappa$-non-collapsing assumption implies, by Hamilton's compactness result [H3], that

$$
\left(\tilde{B}_{1}\left(x(\tau), \sqrt{\frac{1}{\epsilon}}\right) \times\left(\delta, \frac{1}{\delta}\right), \tilde{g}^{\tau}(s)\right) \rightarrow\left(B^{\infty}\left(x_{\infty}, \sqrt{\frac{1}{\epsilon}}\right) \times\left(\delta, \frac{1}{\delta}\right), g^{\infty}(s)\right)
$$

as solutions to Kähler-Ricci flow. This can be extended to an ancient solution $\left(M_{\infty}, g_{\infty}(\tau)\right)$. The estimates (1.15)-(1.17) ensure that $\ell(y, s)$ the reduced distance with respect to re-scaled metric survives under the limit and converges to a function $\ell_{\infty}(y, s)$. Let

$$
V_{\infty}(s)=\int_{M_{\infty}} \frac{e^{-\ell_{\infty}(y, s)}}{s^{\frac{n}{2}}} d \mu_{s}
$$

It was claimed by Perelman in $[\mathrm{P} 1,11.2]$ that

\section{Claim 2.}

$$
\lim _{\tau \rightarrow \infty} \tilde{V}(\tau)=V_{\infty}(s)
$$

In particular, one has that $V_{\infty}(s)$ is a constant and $\left(M_{\infty}, g^{\infty}\right)$ is a non-flat gradient shrinking soliton.

The claim follows if one can show that

$$
\int_{\left(B_{\tau}\left(x(\tau), \sqrt{\frac{\tau}{\epsilon}}\right)\right)^{c}} \frac{e^{-\ell(y, \tau)}}{\tau^{\frac{n}{2}}} d \mu_{\tau} \leq C(\epsilon)
$$

with $C(\epsilon) \rightarrow 0$ as $\epsilon \rightarrow 0$. This can be proved easily if we can get an 'effective' lower bound estimate of $\ell(y, \tau)$ in terms of $\frac{d_{\tau}^{2}\left(x_{0}, y\right)}{\tau}$. This last point was proved in [Ye, Lemma 2.2]. In fact it was proved that there exists positive constant $C=C(n)$ such that for any $y, z \in M$.

$$
-\ell(z, \tau)-1+C \frac{d_{\tau}^{2}(z, y)}{\tau} \leq \ell(y, \tau) .
$$

One should consult notes [KL, Ye] for more detailed exposition on the proof of Claim 2.

Proof of Theorem 2. Assume that $(M, g(t))$ is an ancient solution (non-flat) defined on $M \times(\infty, 0]$. If $M$ is compact, there is nothing to prove. So we assume that $M$ is non-compact. We prove the theorem by the contradiction. So we assume that $\mathcal{V}\left(g\left(t_{0}\right)\right)>0$ for some $t_{0}$. By passing to it universal cover we can also assume that $M$ is simply-connected. Then by part (ii) of Theorem 4.2 of [NT2] again (see also Corollary 4.1 of [NT2]), we have that the scalar curvature 
has the average decay (1.10). Now apply Theorem 2.2 of [NT1] (and its proof for the time before $\left.t_{0}\right)$ we conclude that for all $t, \mathcal{V}(g(t))=\mathcal{V}\left(g\left(t_{0}\right)\right)>0$. This in particular implies that $(M, g(t))$ is $\kappa$-noncollapsed in all scales (since the volume is non-collapsed even without assuming the curvature bound).

Now we apply the above blow-down procedure of Perelman to obtain the limit $\left(M_{\infty}, g^{\infty}\right)$, which is a gradient shrinking soliton. Since $(M, g(t))$ is assumed to have nonnegative bisectional curvature, the limit $\left(M_{\infty}, g^{\infty}\right)$ also has nonnegative bisectional curvature. By the definition of $\left(M_{\infty}, g^{\infty}\right)$, it is clear that the corresponding asymptotic volume ratio $\mathcal{V}\left(M_{\infty}, g^{\infty}\right) \geq \mathcal{V}\left(g\left(t_{0}\right)\right)>0$. On the other hand, since $\left(M_{\infty}, g^{\infty}\right)$ is non-flat, its scalar curvature must be positive by the strong maximum principle. Now we can apply Theorem 3 (part (ii)) to the universal cover of $M_{\infty}$ and conclude that $M_{\infty}$ can not have maximum volume growth. This contradicts the fact that $\mathcal{V}\left(M_{\infty}, g^{\infty}\right) \geq \mathcal{V}\left(g\left(t_{0}\right)\right)>0$. The contradiction proves the theorem.

\section{Applications to Kähler manifolds and Kähler-Ricci flow}

Theorem 1 has nice applications to Ricci flow as shown in [P1]. Following [P1], we can derive the compactness result on ancient solutions to Kähler-Ricci flow out of Theorem 2 .

We call an ancient non-flat solution $(M, g(t))$ defined on $M \times(-\infty, 0]$ a $\kappa$ solution to Kähler-Ricci flow, if $(M, g(t))$ has nonnegative bisectional curvature, satisfying the trace differential Harnack inequality

$$
\mathcal{R}_{t}+\langle\nabla \mathcal{R}, X\rangle+\langle X, \nabla \mathcal{R}\rangle+\operatorname{Ric}(X, X) \geq 0
$$

for any $(1,0)$ vector field $X$, and $(M, g(t))$ is $\kappa$-non-collapsed on all scales for some fixed $\kappa>0$. Recall that $\kappa$-non-collapsed on all scales means that for any time $t$ and $x_{0} \in M$ if for all $y \in B_{t}\left(x_{0}, r\right), \mathcal{R}(y, t) \leq \frac{1}{r^{2}}$, then $\operatorname{Vol}_{t}\left(B_{t}\left(x_{0}, r\right)\right) \geq$ $\kappa r^{2 m}$. Notice that we do not require $\mathcal{R}$ being bounded. We formulate in such way to be able to make the result hold for any dimension.

Theorem 2.1. The set of $\kappa$-solutions to Kähler-Ricci flow is compact modulo scaling.

The proof of the result follows the same line of the argument as Theorem 11.7 of $[\mathrm{P} 1]$. The argument in [P1] is quite robust. The key components of the argument in [P1] are Shi's local derivative estimate, trace differential Harnack and the following consequence of Theorem 2 .

Corollary 2.1. For every $\omega>0$ there exist $B=B(\omega)<\infty, C=C(\omega)<$ $\infty, \tau_{0}=\tau_{0}(\omega)>0$, with the following properties.

(a) Suppose we have a (not necessarily complete) solution $g(t)$ to the KählerRicci flow, defined on $M \times\left[t_{0}, 0\right]$, so that at time $t=0$ the metric ball $B_{0}\left(x_{0}, r_{0}\right)$ is compactly contained in $M$. Suppose that at each time $t, t_{0} \leq t \leq 0$, the metric $g(t)$ has nonnegative bisectional curvature, and $\operatorname{Vol}_{t}\left(B_{t}\left(x_{0}, r_{0}\right)\right) \geq \omega r_{0}^{n}$. Then we have an estimate $R(x, t) \leq C r_{0}^{-2}+B\left(t-t_{0}\right)^{-1}$ whenever $d_{t}\left(x, x_{0}\right) \leq \frac{1}{4} r_{0}$. 
(b) If, rather than assuming a lower bound on volume for all $t$, we assume it only for $t=0$, then the same conclusion holds with $-\tau_{0} r_{0}^{2}$ in place of $t_{0}$, provided that $-t_{0} \geq \tau_{0} r_{0}^{2}$.

The corollary above is exactly the same as Corollary 11.6 of [P1] with only the Ricci flow being replaced by Kähler-Ricci flow and curvature operator being replaced by bisectional curvature. The proof is the also the same if one replaces Theorem 1 of Perelman, whereever it is needed, by Theorem 2 of this paper. The robust scaling argument in the proof of Corollary 11.6 in [P1] also resembles, to some degree in the spirit, various curvature estimates proved by certain scaling argument in the study of the mean curvature flow and other PDEs. See for example, [S1, E, Wh], as well as Simon's proof on Schauder's estimates in [S2]. The detailed exposition on the proof of the above Theorem 2,1 and Corollary 2.1 can be found in [CLN, KL, STW] as well as author's AIM lecture notes.

In view of the compactness result above and the general tensor maximum principle proved in [NT2], we conjecture that H.-D. Cao's matrix Li-Yau-Hamilton estimate holds on any complete Kähler manifolds with non-negative bisectional curvature. No assumption on curvature being bounded is needed. If confirmed, one has Theorem 2.1 for all $\kappa$-noncollapsed ancient solutions with non-negative bisectional curvature. As in [P1], the following gradient estimate is a consequence of Theorem 2.1.

Corollary 2.2. There exists $C=C(\kappa, m)$ such that for the $\kappa$-solution $(M, g(t))$ we have that

$$
|\nabla \mathcal{R}|(x, t) \leq C \mathcal{R}^{\frac{3}{2}}(x, t), \quad\left|\mathcal{R}_{t}\right|(x, t) \leq C \mathcal{R}^{2}(x, t) .
$$

Note that Theorem 2 also holds for ancient solutions with nonnegative bisectional curvature and differential Harnack (2.1). Also notice that the $\kappa$-solution here has different meaning from [P1. Sectiona 11]. The bounded $\kappa$-soltion defined last section corresponds to the $\kappa$-solution in [P1].

Proof of Corollary 1. By Shi's short time existence result we know that the solution exists until the curvature blows up. But by Corollary 2.1 above (see also $[\mathrm{P} 1,11.5$ and 11.6]) one has the estimate

$$
\mathcal{R}(x, t) \leq \frac{C_{1}}{t+1},
$$

for some $C_{1}=C_{1}(n, \mathcal{V}(g(0)))>0$. Therefore, the solution exists for all time and is of Type III. Namely there is no slowly forming singularity at infinity. The result can also be shown using Hamilton's blow-up argument in [H5]. In order to get the curvature decay estimate $(0.2)$ we first apply Theorem 2.1 of [NT1] to conclude that there exists $C_{2}=C_{2}(m)$ with

$$
\int_{0}^{\sqrt{t}} s k(x, s) d s \leq-C_{2} F(x, t)
$$


where $k(x, r)=\int_{B_{x}(r)} \mathcal{R}(y, 0) d \mu$, with respect to the initial metric and

$$
F(x, t)=\log \left[\frac{\operatorname{det}\left(g_{\alpha \bar{\beta}}(x, t)\right)}{\operatorname{det}\left(g_{\alpha \bar{\beta}}(x, 0)\right)}\right] .
$$

(The above estimate follows easily from that facts $-F(x, t) \geq 0$ and $\left(\Delta_{0}-\frac{\partial}{\partial t}\right)(-F)(x, t) \leq-\mathcal{R}(x, 0)$, where $\Delta_{0}$ is the Laplace operator with respect to $g(x, 0)$. This is indeed the proof on page 125 of [NT1].) Since $-\frac{\partial}{\partial t} F=$ $\mathcal{R}(x, t) \leq \frac{C_{1}}{1+t}$, for $1 \ll t$, one has that

$$
\int_{0}^{r} s k(x, s) d s \leq C_{3} \log (r+2)
$$

for some $C_{3}=C_{3}(m, \mathcal{V}(g(0)))$.

Using the curvature decay estimate (2.2) together with the fact that the asymptotic volume ratio $\mathcal{V}(t)$ is a constant function of $t$, one can apply the local injectivity radius estimate of Cheeger-Gromov-Taylor [CGT, Theorem 4.3] (see also [CLY] for earlier similar works) to conclude that the injectivity radius of $(M, g(t))$ has the lower bound $C \sqrt{t}$, where $C$ is a constant independent of $t$. This implies that $M$ can be exhausted by open subdomains which diffeomorphic to Euclidean balls. From this one can conclude the topological type of $M$ from by-now standard result from topology. The above is the observation in [CZ3], which follows the earlier construction in [Sh4, Section 9] and [H6]. Note that the Steinness of $M$ has been proved for any complete Kähler manifolds with the maximum volume growth and nonnegative bisectional curvature in [NT2, Corollary 4.2] (Please see also [WZ] for the even easier positive case.) One then can adapt the construction of [Sh4, Section 9] to conclude that $M$ is biholomorphic to a pseudoconvex domain in $\mathbb{C}^{m}$.

To obtain (0.2), by [NT2, Theorem 6.1], we first solve the Poincaré-Lelong equation to obtain $u$ such that $\partial_{\alpha} \bar{\partial}_{\bar{\beta}} u=R_{\alpha \bar{\beta}}$, and $u$ is at most of logarithmic growth. By considering its heat equation deformation and adding a function of the form $\log \left(|z|^{2}+1\right)$ in the case $M$ splits some factors of $\mathbb{C}$, noticing that $M$ is diffeomorphic to $\mathbb{R}^{n}$, we can obtain a strictly plurisubharmonic function of logarithmic growth on M. More precisely, let $v(x, t)$ be the heat equation deformation of $u(x)$ (without Kähler-Ricci flow). By [NT2], $v(x, t)$ has the same growth as $u(x)$ and $M$ splits as $M=M_{1} \times M_{2}$, where on $M_{1}$ the complex Hessian $v_{\alpha \bar{\beta}}(x, t)$ is positive definite and on $M_{2}, v_{\alpha \bar{\beta}}(x, t) \equiv 0$. By the result of Cheng-Yau we know that $v(x, t)$ must be a constant on $M_{2}$, which then implies that $u(x)$ is a constant on $M_{2}$. This implies that $M_{2}=\mathbb{C}^{k}$ for some $0 \leq k \leq m$. On $M_{2}$ one can construct a strictly plurisubharmonic function of logarithmic growth by taking $\phi(z)=\log \left(|z|^{2}+1\right)$. Adding this to $v(x, t)$ we have a strictly plurisubharmonic function of logarithmic growth on $M$. Now (0.2) follows from the proof of Corollary 3.2 in [N2].

Proof of Corollary 2. By the above proof of Corollary 1 we know that $M$ supports a strictly plurisubharmonic function of logarithmic growth. The conclusion on 
the transcendence degree of rational function field follows from the $L^{2}$-estimate of $\bar{\partial}$ and the dimension estimate proved in Theorem 3.1 of [N2]. See, for example, [N2, Theorem 5.2] for the constructions of holomorphic functions of polynomial growth, forming a local holomorphic coordinate for any given point in $M$. The construction via the well-known Hörmander's $L^{2}$-estimates provides the existence of plenty holomorphic functions of polynomial growth, which provides a lower bound on the transcendence degree. The multiplicity estimates in Theorem 3.1 of [N2] gives the upper bound on the transcendental elements of the rational functions. The assertion that $M$ is an affine quasi-algebraic variety follows from the construction in [M1]. See also [De] as well as [CTZ]. Notice that we now also have uniform multiplicity estimates, thanks to the new monotonicity formula and Theorem 3.1 in [N2], which simplifies the steps of [M1] quite a bit. Here by an affine quasi-algebraic variety we mean an affine algebraic variety with some codimension one algebraic varieties removed.

The part $M$ is biholomorphic to $\mathbb{C}^{2}$ also follows as in [M1, page 256] by appealing to the result of Ramanujam that any quasi-projective surface homeomorphic to $\mathbb{R}^{4}$ is biholomorphic to $\mathbb{C}^{2}$.

Remark 2.1. The fact that maximum volume growth implies the ampleness of holomorphic functions of polynomial growth was conjectured in [N2]. It has been shown in [NT2] that the positivity of Ricci curvature together with some average curvature decay assumption also implies the same result on holomorphic function of polynomial growth. In fact, this also implies that $M$ is an affine quasi-algebraic variety as in Corollary 2.

The existence of harmonic functions of polynomial growth was obtained in [D] under the assumption of nonnegative Ricci curvature, maximum volume growth and the uniqueness of the tangent cone at infinity. The result here seems to indicate that the assumption on the uniqueness of the tangent cone may not be needed.

After the completion of current paper, H.-D. Cao informed the author that the part of result in Corollary 2, on the special case that the complex dimension $m=2$, assuming the maximum volume growth and nonnegativity of bisectional curvature, was also obtained by B.-L. Chen earlier in his thesis.

\section{Applications to Ricci flow}

The proof of Proposition 1.1 can be used in some other situations. For example we can prove the following results.

\section{Proposition 3.1.}

(i) Let $(M, g)$ be a compete steady gradient soliton. Assume that the Ricci curvature is pinched in the sense that $R_{i j} \geq \epsilon \mathcal{R} g_{i j}$, for some $\epsilon>0$ with the scaler curvature $\mathcal{R}(x)>0$. Then there exist $C>0, a>0$ such that

$$
\mathcal{R}(x) \leq C \exp (-a(r(x)+1))
$$


Here $r(x)$ is the distance function to some fixed point in $M$.

(ii) Let $(M, g)$ be a compete expanding gradient soliton. Assume that the Ricci curvature is pinched as above. Then (3.1) holds.

Corollary 3.1. Assume that $n \geq 3$.

(i) There is no steady gradient soliton with pinched Ricci curvature as in Proposition 3.1;

(ii) There is no expanding gradient soliton with pinched Ricci curvature (as in Proposition 3.1) and nonnegative sectional curvature.

In particular, any non-flat complete three manifolds with pinched Ricci curvature and bounded nonnegative sectional curvature must be compact, therefore spherical. Same result holds for any higher dimensional complete Riemannian manifolds with pinched curvature operator in the sense that

$$
|\stackrel{\circ}{R} m|^{2}=|W|^{2}+|V|^{2} \leq \delta_{n}(1-\epsilon)^{2}|U|^{2}=\delta_{n}(1-\epsilon)^{2} \frac{2}{n(n-1)} \mathcal{R}^{2}
$$

where $\epsilon>0, \delta_{3}>0, \delta_{4}=\frac{1}{5}, \delta_{5}=\frac{1}{10}$ and $\delta_{n}=\frac{2}{(n-2)(n+1)}$, and $W, V$ and $U$ denote the Weyl curvature tensor, traceless Ricci part and the scalar curvature part, respectively, according to the curvature operator decomposition in [Hu].

Proof. The first part follows from Theorem 20.2 of [H5] and (i) of Proposition 3.1. Notice that the proof there works under the weaker assumption $R_{i j}>0$.

For part (ii), one just needs to recall the gap theorem of Greene-Wu [GW] (see also $[\mathrm{PT}]$ ) asserting that a simply-connected complete Riemannian manifold with nonnegative sectional curvature and (3.1) must be flat, noticing that under the assumption of expanding gradient soliton and nonnegativity of the Ricci curvature, $M$ is diffeomorphic to $\mathbb{R}^{n}$ (which in particular implies that the new expanders examples constructed in $[\mathrm{FIK}]$ can not have nonnegative Ricci curvature), since $f$ is a strictly convex function with only one critical point.

By (i) and (ii), the singularity analysis of Ricci flow implies that curvature pinched manifolds must be compact. This is the main result proved in [CZ1]. Now the last claim in the corollary just restates the fundamental results of Hamilton [H1] and Huisken [Hu].

Note that when $n=2,(3.1)$ is automatic and the examples of Hamilton's cigar and the expanding soliton exhibited in [CLN] show that the exponential decay proved in Proposition 3.1 is sharp.

Remark 3.1. We speculate that there is no complete noncompact Riemannian manifold with the pinched Ricci as in Proposition 3.1 (according to B. Chow, this was speculated earlier, in the case that $n=3$, by $R$. Hamilton). If true, this should be the right Bonnet-Meyers type theorem since that (3.2) is too strong to allow any other topology. Due to the page limit, the details on the proof of results in this section will appear somewhere else. 


\section{Acknowledgements}

The author would like to thank Professors B. Chow, T. Ilmanen, R. Schoen and Jon Wolfson for helpful discussions, Professor P. Lu for pointing out the reference [Ye], Professors X. Chen, L.-F. Tam, M.-T. Wang, H. Wu and F.-Y. Zheng for their interests. Professor H.-D. Cao pointed out to the author that the earlier version of Corollary 2 has further complex geometric consequences relating to the uniformization of Kähler manifolds with nonnegative bisectional curvature. It is a pleasure to record our gratitude to him. Finally we would like to thank the referee for pointing out a discrepancy in the earlier version of this paper (of which the author was also aware independently).

\section{References}

[B] S. Bando, On classification of three-dimensional compact Kähler manifolds of nonnegative bisectional curvature, J. Differential Geom. 19 (1984), 283-297.

[Br] R. Bryant, Gradient Kähler-Ricci solitons, preprint.

[C1] H.-D. Cao, Deformation of Kähler metrics to Kähler-Einstein metrics on compact Kähler manifolds, Invent. Math. 81 (1985), 359-372.

[C2] - On Harnack's inequalities for the Kähler-Ricci flow, Invent. Math. 109 (1992), 247-263.

[C3] L Limits of solutions to Kähler-Ricci flow, J. Differential. Geom. 45 (1997), 257-272.

[C4] On dimension reduction in Kähler-Ricci flow, Comm. Anal. Geom. 12 (2004), 305-320.

[CC] H.-D. Cao and B. Chow, Recent developments on the Ricci flow, Bull. Amer. Math. Soc. 36 (1999), 59-74.

[CT1] A. Chau and L.-F. Tam, A note on the uniformization of gradient Kähler-Ricci solitons, Math. Res. Lett. 12 (2005), 19-21.

[CT2] — On the complex structure of Kähler manifolds with nonnegative curvature, preprint.

[CGT] J. Cheeger, M. Gromov and M. Taylor, Finite propagation speed, kernel estimates for functions of the Laplace operator, and the geometry of complete Riemannian manifolds, J. Differential Geom. 17 (1982), no. 1, 15-53.

[Cn] X.-X. Chen, Recent progress in Kähler geometry, Proccedings of the ICM II (2002), Higher Ed. Press, Beijing, 2002, 273-282.

[CZ1] B.-L. Chen and X.-P. Zhu, Complete Riemannian manifolds with pointwise pinched curvature, Invent. Math. 140 (2000), no. 2, 423-452.

[CZ2] Zhu, A property of Kähler-Ricci solitons on complete complex surfaces, Geometry and Nonlinear PDE, AMS/IP Studies in Advanced Mathematics 29 (2002), $5-12$.

[CZ3] On complete noncompact Kähler manifolds with positive bisectional curvature, Math. Ann. to appear.

[CZ4] - Volume growth and curvature decay of positively curved Kähler manifolds, preprint.

[CTZ] B.-L. Chen, S.-H. Tang and X.-P. Zhu, A uniformization theorem of complete noncompact Kähler surfaces with positive bisectional curvature, arXiv:math.DG/0211372.

[CLY] S.-Y. Cheng, P. Li and S.-T. Yau, On the upper estimate of the heat kernel of a complete Riemannian manifold, Amer. J. Math. 103 (1981), no. 5, 1021-1063.

[Ch] B. Chow, The Ricci flow on the 2-sphere, J. Differential Geom. 33 (1991), 325-334.

[CK] B. Chow and D. Knopf, The Ricci flow, vol. I: an introduction, Math. Surveys and Monographs, AMS., Vol. 110, 2004. 
[CLN] B. Chow, P. Lu and L. Ni, Hamilton's Ricci flow, in preparation.

[Cu] S.-C. Chu, Geometry of 3-dimensional gradient Ricci solitons with positive curvature, preprint.

[De] J.-P. Demailly, Mesures de Monge-Ampère et caractérisation géométrique des variétés algébri -ques affines, Mm. Soc. Math. France (N.S.) 19 (1985), 1-125.

[D] Y. Ding, An existence theorem of harmonic functions with polynomial growth, Proc. Amer. Math. Soc. 132 (2004), no. 2, 543-551.

[E] K. Ecker, Regularity theory for mean curvature flow. Progress in Nonlinear Differential Equations and their Applications, 57. Birkhuser Boston, Inc., Boston, MA, 2004.

[FIK] M. Feldman, T. Ilmanen and D. Knopf, Rotationally symmetric shrinking and expanding gradient Kähler-Ricci solitons, J. Differential Geom. 65 (2003), no. 2, 169-209.

[GW] R. Greene and H. Wu, Gap theorems for noncompact Riemannian manifolds, Duke Math. J. 49 (1982), no. 3, 731-756.

[H1] R. S. Hamilton, Three-manifolds with positive Ricci curvature, J. Differential Geom. 17 (1982), no. 2, 255-306.

[H2] , Four-manifolds with positive curvature operator, J. Differential Geom. 24 (1986), no. 2, 153-179.

[H3] , The Ricci flow on surfaces, Contemp. Math. 71 (1988), 237-261.

[H4] Eternal solutions to the Ricci flow, J. Differential Geom. 38 (1993), no. 1, $1-11$.

[H5] Formation of singularities in the Ricci flow, Surveys in Diff. Geom. 2 (1995), $7-136$.

[H6] A compactness property for solutions of Ricci flow, Amer. Jour. Math. 117 (1995), 545-572.

[H7] Convex hypersurfaces with pinched second fundamental form, Comm. Anal. Geom. 2 (1994), no. 1, 167-172.

[Hu] G. Huisken, Ricci deformation of the metric on a Riemannian manifold, J. Differential Geom. 21 (1985), no. 1, 47-62.

[I] T. Ivey, Ricci solitons on compact Kähler surfaces, Proceedings of AMS. 125 (1997), 1203-1208.

[KL] B. Kleiner and J. Lott, Notes on Perelman's paper.

[Ko] N. Koiso, On rotationally symmetric Hamilton's equation for Kähler-Einstein metrics, Adv. Studies in Pure Math. 18 (1990), Recent Topics in Differential and Analytic Geometry pp. 327-337.

[LW] P. Li and J.-P. Wang, Hölder estimates and regularity for holomorphic and harmonic functions, J. Differential Geom. 58 (2001), no. 2, 309-329.

[M1] N. Mok, An embedding theorem of complete Kähler manifolds of positive bisectional curvature onto affine algebraic varieties, Bull. Soc. Math. France 112 (1984), no. 2, 197-250.

[M2] - The uniformization theorem for compact Kähler manifolds of nonnegative holomorphic bisectional curvature, J. Differential Geom. 27 (1988), 179-214.

[M3] _ An embedding theorem of complete Kähler manifolds of positive Ricci curvature onto quasi-projective varieties, Math. Ann. 286 (1990), 373-408.

[M4] , Topics in complex differential geometry, Adv. Studies in Pure Math. 18 (1990), 1-141.

[N1] L. Ni, Monotonicity and Kähler-Ricci flow, Proceedings of the 2002 Workshop on Geometric Evolution Equations, Contemp. Math. 367 (2005), 149-165.

[N2] , A monotonicity formula on complete Kähler manifolds with nonnegative bisectional curvature, J. Amer. Math. Soc. 17 (2004), 909-946.

[N3] - Details of Perelman's work on Kähler-Ricci flow, research notes.

[NST] L. Ni, Y.-G. Shi and L.-F.Tam, Poisson equation, Poincaré-Lelong equation and curvature decay on complete Kähler manifolds, J. Differential Geom. 57 (2001), 339-388. 
[NT1] L. Ni and L.-F.Tam, Kähler Ricci flow and Poincaré-Lelong equation, Comm. Anal. Geom. 12 (2004), 111-141.

[NT2] , Plurisubharmonic functions and the structure of complete Kähler manifolds with nonnegative curvature, J. Differential Geom. 64 (2003), 457-524.

[P1] G. Perelman, The entropy formula for the Ricci flow and its geometric applications, arXiv: math.DG/ 0211159.

[P2] - Ricci flow with surgery on three-manifolds, arXiv: math.DG/ 0303109.

[P3] Talks and informal discussions at MIT and SUNY, Stony Brooks.

[PT] A. Petrunin and W. Tuschmann, Asymptotical flatness and cone structure at infinity, Math. Ann. 321 (2001), no. 4, 775-788.

[STW] N. Sesum, G. Tian and X. Wang, Notes on Perelman's paper.

[Sh1] W. X. Shi, Deforming the metric on complete Riemannian manifolds, J. Differential Geom. 30 (1989), 223-301.

[Sh2] , Ricci deformation of the metric on complete noncompact Riemannian manifolds, J. Differential Geom. 30 (1989), 303-394.

[Sh3] , Ricci deformation of metric on complete noncompact Kähler manifolds, $\mathrm{Ph}$. D. thesis at Harvard University, 1990.

[Sh4] , Ricci flow and the uniformization on complete noncompact Kähler manifolds, J. Differential Geom. 45 (1997), 94-220.

[S1] L. Simon, Remarks on curvature estimates for minimal hypersurfaces, Duke Math. J. 43 (1976), 545-553.

[S2] _ Schauder estimates by scaling, Calc. Var. PDE 5 (1997), 391-407.

[SiY] Y.-T. Siu and S.-T. Yau, Compact Kähler manifolds of positive bisectional curvature, Invent. Math. 59 (1980), no. 2, 189-204.

[St] J. Stallings, The piecewise-linear structure of Euclidean space, Proc. Cambridge Philos. Soc. 58 (1962), 481-488.

[T] G. Tian, Compactness theorems for Kähler-Einstein manifolds of dimension 3 and up, J. Differential Geom. 35 (1992), 535-558.

[Wh] B. White, A local regularity theorem for mean curvature flow, preprint.

[WZ] B. Wong and Q. Zhang, Refined gradient bounds, Poisson equations and some applications to open Kähler manifolds, Asian J. Math. 7 (2003), 337-364.

[WuZ] H. Wu and F.-Y. Zheng, Examples of positively curved complete Kähler manifolds, preprint.

[Y] S.-T. Yau, A review of complex differential geometry, Proc. Symp. Pure Math. 52 (1991), 619-625.

[Ye] R. Ye, Notes on reduced volume and asymptotic Ricci solitons of $\kappa$-solutions, available at http://www.math.lsa.umich.edu/research/ricciflow/perelman.html.

Department of Mathematics, University of California, San Diego, CA 92093

E-mail address: Ini@math.ucsd.edu 